\title{
Are we ready for the ERAS protocol in colorectal surgery?
}

\author{
Michał Kisielewski, Mateusz Rubinkiewicz, Michał Pędziwiatr, Magdalena Pisarska, Marcin Migaczewski, \\ Marcin Dembiński, Piotr Major, Kazimierz Rembiasz, Andrzej Budzyński \\ Second Department of General Surgery, Jagiellonian University Medical College, Krakow, Poland
}

Videosurgery Miniinv 2017; 12 (1): 7-12

DOI: https://doi.org/10.5114/wiitm.2017.66672

\begin{abstract}
Introduction: Modern perioperative care principles in elective colorectal surgery have already been established by international surgical authorities. Nevertheless, barriers to the introduction of routine evidence-based clinical care and changing dogmas still exist. One of the factors is the surgeon.

Aim: To assess perioperative care trends in elective colorectal surgery among general surgery consultants in surgical departments in Malopolska Voivodeship, Poland.

Material and methods: An anonymous standardized 20-question questionnaire was developed based on ERAS principles and sent out to Malopolska Voivodeship general surgery departments. Answers of general surgery consultants showed the level of acceptance of elements of perioperative care.

Results: The overall response rate was 66\%. Several elements (antibiotic and antithrombotic prophylaxis, postoperative oxygen therapy, no nasogastric tubes) had quite a high acceptance rate. On the other hand, most crucial surgical perioperative elements (lack of mechanical bowel preparation, preoperative oral carbohydrate loading, use of laparoscopy and lack of drains, early fluid and oral diet intake, early mobilization) were not followed according to evidence-based ERAS protocol recommendations. Surgeons were not willing to change their practice, but were supportive of changes in anesthesiologist-dependent elements of perioperative care, such as restrictive fluid therapy, use of transversus abdominis plane blocks, etc.

Conclusions: Many elements of perioperative care in elective colorectal surgery in Malopolska Voivodeship are still dictated by dogma and are not evidence-based. The level of acceptance of many important ERAS protocol elements is low. Surgeons are ready to accept only changes that do not interfere with their practice.
\end{abstract}

Key words: surgeons, perioperative management, Polish survey, laparoscopic colorectal surgery.

\section{Introduction}

Modern evidence-based perioperative care principles in elective colorectal surgery have already been established by international surgical authorities [1, 2]. The main ideas include optimization of the patient's preoperative state, use of minimally invasive surgical techniques, balanced intravenous fluid therapy, multimodal non-opioid analgesia, and early mobilization and nutrition [3, 4]. These and other elements work in synergy and aim at reduction of sur- gically induced physiological and metabolic stress. Therefore patients experience fewer perioperative complications, and the length of hospital stay (LOS) is significantly shorter without a negative impact on the readmission rate $[5,6]$. This can be applied in various branches of surgery [7-9]. Guidelines for perioperative care in elective colonic/rectal surgery included in Enhanced Recovery After Surgery (ERAS) Society recommendations are widely available [1, 2]. Nevertheless, barriers to the introduction of routine

\section{Address for correspondence}

Michał Kisielewski MD, Second Department of General Surgery, Jagiellonian University Medical College, 21 Kopernika St, $31-501$ Krakow, Poland, phone: +48 889767 589, e-mail: kisialeuskim@gmail.com 
evidence-based clinical care and changing dogmas still exist. Among potential factors that can impair implementation of the ERAS protocol are staff-related problems $[10,11]$.

\section{Aim}

The aim of the study was to assess perioperative care trends in elective colorectal surgery among general surgery consultants in surgical departments in Malopolska Voivodeship, Poland, where colorectal procedures are routinely performed.

\section{Material and methods}

A questionnaire study was conducted in the period between January and May 2016. An anonymous standardized questionnaire was developed based on ERAS principles and included 20 questions - 14 closed questions with two options to choose, and 6 open questions, where respondents could put in a number, representing for instance length of antibiotic administration (Table I). Questionnaires were approved and supported by Malopolska Voivodeship Chief Consultant in General Surgery, and distributed by mail among all surgical departments in Malopolska Voivodeship in Poland. Only general surgery consultants were asked to participate in the study. Filled out questionnaires were sent back to the authors and then analyzed. To maximize the response to the postal questionnaire a reminder letter was sent to the surgical departments that had a poor feedback rate.
During analysis of acceptance of perioperative care elements, $>75 \%$ was chosen as a level of high acceptance.

\section{Results}

Two hundred and forty-eight questionnaires were sent out; 164 filled in versions from 25 out of 32 surgical departments from Malopolska Voivodeship were received back and then analyzed (overall response rate $66 \%$ ). Depending on level of acceptance, all perioperative elements from questionnaire were divided into two groups - group 1 consisted of elements with a high level of acceptance and group 2 included poorly accepted elements (Table II).

Nearly $87 \%$ of surgeons do discuss individually with their patients what the reconvalescence period will be like. Ninety-six percent of patients will receive antithrombotic and antibiotic prophylaxis, and antibiotics would not be continued postoperatively in $28 \%$ of cases (mean length of antibiotic administration 2.06 days, range: 0-7 days). In 80\% of patients restricted perioperative intravenous fluid therapy will be used. In $84 \%$ of cases the nasogastric tube will be removed shortly after surgery. Postoperative pain management is based on opioid drugs in $73 \%$ of patients, and in $78 \%$ of cases additional locoregional anesthetic techniques would be used. Seventy-nine percent of patients would receive postoperative oxygen therapy.

Thirty percent of surgeons do not use mechanical bowel preparation in colonic surgery, and 20\%

Table I. Elements of perioperative care covered in standardized questionnaire

\begin{tabular}{|llll|}
\hline 1. Preoperative patient education & $\mathrm{Y} / \mathrm{N}^{\wedge}$ & 11. Routine use of drains & $\mathrm{Y} / \mathrm{N}$ \\
\hline 2. Mechanical bowel preparation in colon surgery & $\mathrm{Y} / \mathrm{N}$ & 12. Length of peritoneal drainage & $\mathrm{Y} / \mathrm{N}$ \\
\hline 3. Mechanical bowel preparation in rectal surgery & $\mathrm{Y} / \mathrm{N}$ & 13. Opioid drug use & $\mathrm{Y} / \mathrm{N}$ \\
\hline 4. Preoperative oral carbohydrate loading & $\mathrm{Y} / \mathrm{N}$ & $\begin{array}{l}\text { 14. Use of locoregional analgesia techniques } \\
\text { (TAP block, epidural analgesia) }\end{array}$ & $\mathrm{Y} / \mathrm{N}$ \\
\hline 5. Antithrombotic prophylaxis & $\mathrm{Y} / \mathrm{N}$ & 15. Postoperative nausea and vomiting prophylaxis use & $\mathrm{Y} / \mathrm{N}$ \\
\hline 6. Antibiotic prophylaxis & $\mathrm{Y} / \mathrm{N}$ & 16. Postoperative oxygenation & \\
\hline 7. Length of perioperative antibiotics administration & & 17. Day of oral fluids introduction & \\
\hline 8. Open or laparoscopic surgical approach preferred & & 18. Day of oral solid diet introduction & \\
\hline 9. Restrictive intravenous fluid therapy & $\mathrm{Y} / \mathrm{N}$ & 19. Day of urinary catheter removal \\
\hline 10. Use of nasogastric tube & $\mathrm{Y} / \mathrm{N}$ & 20. Day of mobilization \\
\hline
\end{tabular}

${ }^{\wedge} / N$ - stands for YES/NO answer. Open questions written in Italics. 
Table II. Acceptance of elements of perioperative care according to the questionnaire

\begin{tabular}{|c|c|c|c|}
\hline \multicolumn{2}{|c|}{ Group 1. Highly accepted elements of perioperative care } & \multicolumn{2}{|c|}{ Group 2. Weakly accepted elements of perioperative care } \\
\hline Element & $\%$ of acceptance & Element & $\%$ of acceptance \\
\hline Preoperative patient education & 87 & No bowel preparation, colon & 30 \\
\hline Antithrombotic prophylaxis & 96 & No bowel preparation, rectum & 20 \\
\hline Antibiotic prophylaxis & 96 & Preoperative oral carbohydrate loading & 20 \\
\hline Restrictive intravenous fluid therapy & 80 & Use of laparoscopic techniques & 16 \\
\hline No use of nasogastric tubes & 84 & No routine use of drains & 13 \\
\hline $\begin{array}{l}\text { Use of locoregional techniques for } \\
\text { analgesia }\end{array}$ & 78 & Non-opioid analgesia & 27 \\
\hline Postoperative oxygenation & 79 & $\begin{array}{l}\text { Prophylaxis of postoperative nausea } \\
\text { and vomiting }\end{array}$ & 42 \\
\hline & & Early oral fluid intake & 11 \\
\hline & & Early oral food intake & 2 \\
\hline & & Early urinary catheter removal & 3 \\
\hline & & Early mobilization & 16 \\
\hline
\end{tabular}

do not prepare the patient's large bowel in case of rectal surgery. Only $20 \%$ of patients would receive preoperative oral carbohydrate loading. Sixteen percent of respondents from 5 surgical departments prefer the laparoscopic approach. Thirty percent of patients will have no drains left routinely in peritoneal cavity, and when drains are used the average length is 2.5 days (range: 0-6 days). Prophylaxis of postoperative nausea and vomiting (PONV) would be initiated in $42 \%$ of patients. Early oral fluids and diet would be introduced in $11 \%$ and $2 \%$ of patients subsequently. The urinary catheter would be removed during $24 \mathrm{~h}$ postoperatively in 3\% of patients. Early mobilization would be encouraged in $16 \%$ of patients. Table III presents answers to open questions.

\section{Discussion}

In the analyzed material several elements of perioperative care have quite a high level of acceptance among general surgery consultants (e.g. patient education, antibiotic and antithrombotic prophylaxis, postoperative oxygenation). Nevertheless, a number of crucial elements of modern evidence-based clinical practice are not routinely followed (no bowel preparation, use of laparoscopy, no drains, early oral intake and early mobilization).

It was remarkable that when certain elements were dependent on the surgeon (laparoscopy, no drains, etc) they were very poorly accepted. On the other hand, surgeons were ready to accept elements managed by anesthesiologists (e.g. restrictive intravenous fluid therapy, multimodal analgesia). Inter-

Table III. Open question results

\begin{tabular}{|c|c|c|c|c|c|}
\hline Parameter assessed in open question & $<24 \mathrm{~h}$ & $1^{\text {st }}$ day & $2^{\text {nd }}$ day & $3^{\text {rd }}$ day & $\geq 4^{\text {th }}$ day \\
\hline Length of antibiotic prophylaxis & $28 \%$ & $21 \%$ & $9 \%$ & $22 \%$ & $20 \%$ \\
\hline Length of peritoneal drainage & $10 \%$ & $5 \%$ & $32 \%$ & $25 \%$ & $28 \%$ \\
\hline Introduction of oral fluids & $11 \%$ & $50 \%$ & $26 \%$ & $9 \%$ & $4 \%$ \\
\hline Introduction of oral diet & $2 \%$ & $10 \%$ & $32 \%$ & $36 \%$ & $20 \%$ \\
\hline Removal of urinary catheter & $3 \%$ & $33 \%$ & $24 \%$ & $26 \%$ & $14 \%$ \\
\hline Mobilization of patient & $16 \%$ & $65 \%$ & $14 \%$ & $4 \%$ & $1 \%$ \\
\hline
\end{tabular}


estingly, an ERAS survey among anesthesiologists from 27 countries by Greco et al. showed low acceptance of most anesthesiological elements (avoidance of premedication and opioids, targeted intravenous fluid policy, preoperative fasting and early nasogastric tube removal) [12].

\section{Lack of mechanical bowel preparation}

Compared to results from Malopolska Voivodeship, even a smaller percentage of Spanish (14\%) and Swedish (3\%) colorectal surgeons do not prepare the bowel for elective resections $[13,14]$. According to an available Cochrane meta-analysis there is absolutely no benefit from bowel preparation in every available context (amount of anastomotic leakage, wound infections, reoperations, mortality, etc.), and it should be reserved for selected cases [15].

\section{Preoperative oral carbohydrate loading}

An element that is not accepted by surgeons not only in southern Poland but also in western surgical centers is preoperative oral carbohydrate loading [16]. In Spain only 3\% of surgeons use it. Traditional preoperative fasting results in increased insulin resistance, causes longer postoperative ileus and prolongs LOS [13]. The preoperative oral carbohydrate loading can decrease these negative outcomes prior to elective colorectal surgery in a safe manner $[17,18]$.

\section{Laparoscopy}

There is plentiful evidence that laparoscopy short- and long-term outcomes are very good [1921]. When combined with the ERAS protocol, laparoscopy shows the best treatment outcomes and therefore should be strongly promoted among surgeons around the globe [22]. Nevertheless, barriers to laparoscopy in colorectal surgery are still numerous [23]. Among the most common are lack of skills and adequate training. Moreover, some believe that laparoscopy is associated with higher costs and can be oncologically inferior to open surgery [21, 24]. This requires actions from health education managers directed at widespread application of laparoscopic techniques. Interestingly, a survey from the UK showed that laparoscopy enthusiasts are good at implementing other elements of the ERAS protocol [25].

\section{Drains}

In uncomplicated elective colorectal surgery they do not offer lower morbidity and mortality, and do not influence the number of anastomotic leakages, and hence should be discouraged [26-28]. Still, as seen in an Austrian and Germany survey, too many surgeons still leave drains [29].

\section{Early catheter removal}

Very low acceptance is also seen with early urinary catheter removal - only $3 \%$ of respondents would remove it within the first $24 \mathrm{~h}$, despite the fact that it is safe and does not increase the risk of urinary retention $[1,2]$.

\section{Early mobilization}

This is known to be one of the key elements of perioperative care allowing faster recovery [30]. A problem with early mobilization could result from low compliance with other elements, such as prolonged catheterization and routine drain use. Therefore change of perioperative care of the majority of respondents should be encouraged, since the acceptance rate of early mobilization was only $16 \%$.

\section{Postoperative nil per os diet}

This also has no scientific basis. Lewis et al. after a meta-analysis of 13 trials concluded that early enteral nutrition is associated with reduced mortality and may be of significant benefit [31]. In a British survey $88 \%$ and $80 \%$ of patients were allowed fluids and solid food intake on the first postoperative day, respectively [25].

\section{Limitations of the study}

This study shows the view of the general surgery consultants rather than colorectal surgeons as in other similar studies. Data were collected from only one region of Poland. The response rate was average, but comparable to other questionnaire studies on the topic. Acceptance of certain elements of perioperative care does not mean they are routinely practiced by the respondent.

\section{Conclusions}

Many elements of perioperative care in elective colorectal surgery in Malopolska Voivodeship are 
still dictated by dogma and are not evidence-based. The level of acceptance of many important ERAS protocol elements is low. Surgeons are ready to accept only changes that do not interfere with their practice. A barrier resulting from unwillingness to change surgeon-dependent elements still exists. Workshops, seminars and constant internal and/or external audits may increase the acceptance of modern evidence-based perioperative care. Moreover, laparoscopic colorectal resections should become more available, even though ERAS protocol elements can also be used in open surgery.

\section{Acknowledgments}

The publication of this article was supported by the Faculty of Medicine, Jagiellonian University Medical College.

\section{Conflict of interest}

The authors declare no conflict of interest.

\section{References}

1. Gustafsson UO, Scott MJ, Schwenk W, et al. Guidelines for perioperative care in elective colonic surgery: Enhanced Recovery After Surgery (ERAS $\left.{ }^{\circledR}\right)$ Society recommendations. Clin Nutr 2012; 31: 783-800.

2. Nygren J, Thacker J, Carli F, et al. Guidelines for perioperative care in elective rectal/pelvic surgery: Enhanced Recovery After Surgery (ERAS ${ }^{\circledR}$ ) Society recommendations. World I Surg 2013; 37: 285-305.

3. Lassen K, Soop M, Nygren J, et al. Consensus review of optimal perioperative care in colorectal surgery: Enhanced Recovery Af ter Surgery (ERAS) Group recommendations. Arch Surg 2009; 144: 961-9.

4. Pędziwiatr M, Kisialeuski M, Pisarska M, et al. Early implementation of Enhanced Recovery After Surgery (ERAS ${ }^{\circledR}$ ) protocol compliance improves outcomes: a prospective cohort study. Int J Surg 2015; 21: 75-81.

5. Kehlet H. Fast-track colorectal surgery. Lancet 2008; 371: 791-3.

6. Zhuang CL, Ye XZ, Zhang XD, et al. Enhanced recovery after surgery programs versus traditional care for colorectal surgery: a meta-analysis of randomized controlled trials. Dis Colon Rectum 2013; 56: 667-78.

7. Pędziwiatr M, Matłok M, Kisialeuski M, et al. Enhanced recovery (ERAS) protocol in patients undergoing laparoscopic total gastrectomy. Videosurgery Miniinv 2014; 9: 252-7.

8. Małczak P, Pisarska M, Major P, et al. Enhanced recovery after bariatric surgery: systematic review and meta-analysis. Obes Surg 2017; 27: 226-35.

9. Pędziwiatr M, Pisarska M, Kisielewski M, et al. Is ERAS in laparoscopic surgery for colorectal cancer changing risk factors for delayed recovery? Med Oncology (Northwood) 2016; 33: 3-25.
10. Lyon A, Solomon MJ, Harrison JD. A qualitative study assessing the barriers to implementation of enhanced recovery after surgery. World I Surg 2014; 38: 1374-80.

11. Pearsall EA, Meghji Z, Pitzul KB, et al. A qualitative study to understand the barriers and enablers in implementing an enhanced recovery after surgery program. Ann Surg 2015; 261: 92-6.

12. Greco M, Gemma M, Braga M, et al. Enhanced recovery after surgery - a survey among anesthesiologists from 27 countries. Eur J Anaesthesiol 2014; 31: 285-91.

13. Roig JV, Garcia-Fadrique A, Redondo C, et al. Perioperative care in colorectal surgery: current practice patterns and opinions. Colorectal Dis 2009; 11: 976-83.

14. Lassen K, Hannerman P, Ljunqvist O, et al. Patterns in current perioperative practice: survey of colorectal surgeons in five northern European countries. BMJ 2005; 18: 1420-1.

15. Güenaga KF, Matos D, Wille-Jørgensen P. Mechanical bowel preparation for elective colorectal surgery. Cochrane Database Syst Rev 2011; 9: CD001544.

16. Ljungqvist O. Insulin resistance and outcomes in surgery. J Clin Endocrinol Metabol 2010; 95: 4217-9.

17. Nygren J, Thorell A, Ljungqvist O. Preoperative oral carbohydrate therapy. Curr Opin Anaesthesiol 2015; 28: 364-9.

18. Jones C, Badger SA, Hannon R. The role of carbohydrate drinks in preoperative nutrition for elective colorectal surgery. Ann R Coll Surg Engl 2011; 93: 504-7.

19. McKay GD, Morgan MJ, Wong SK, et al. Improved short-term outcomes of laparoscopic versus open resection for colon and rectal cancer in an area health service: a multicenter study. Dis Colon Rectum 2012; 55: 42-50.

20. Guillou PJ, Quirke P, Thorpe H, et al. Short-term endpoints of conventional versus laparoscopic-assisted surgery in patients with colorectal cancer (MRC CLASICC trial): multicenter, randomized controlled trial. Lancet 2005; 365: 1718-26.

21. Kuhry E, Schwenk W, Gaupset R, et al. Long-term results of laparoscopic colorectal cancer resection. Cochrane Database Syst Rev 2008; 2: CD003432.

22. Li MZ, Xiao LB, Wu WH, et al. Meta-analysis of laparoscopic versus open colorectal surgery within fast-track perioperative care. Dis Colon Rectum 2012; 55: 821-7.

23. Kahokehr A, Robertson P, Sammour T, et al. Perioperative care: a survey of New Zealand and Australian colorectal surgeons. Colorectal Dis 2011; 13: 1308-13.

24. Pędziwiatr $M$, Wierdak $M$, Nowakowski $M$, et al. Cost minimization analysis of laparoscopic surgery for colorectal cancer within the enhanced recovery after surgery (ERAS) protocol: a single-center, case-matched study. Videosurgery Miniinv 2016; 11: 14-21.

25. Arsalani-Zadeh R, Ullah S, Khan S, et al. Current pattern of perioperative practice in elective colorectal surgery; a questionnaire survey of ACPGBI members. Int J Surg 2010; 8: 294-8.

26. Jesus E, Karliczek A, Matos D, et al. Prophylactic anastomotic drainage for colorectal surgery. Cochrane Database Syst Rev 2004; 18: CD002100.

27. Bretagnol F, Slim K, Faucheron JL. Anterior resection with low colorectal anastomosis. To drain or not? Ann Chir 2005; 130: 336-9. 
28. Kisialeuski M, Pędziwiatr M, Matłok M. Enhanced recovery after colorectal surgery in elderly patients. Videosurgery Miniinv 2015; 10: 30-6.

29. Hassenberg T, Keese M, Langle F, et al. Fast-track colonic surgery in Austria and Germany - results from the survey on patterns in current perioperative practice. Colorectal Dis 2009; 11: 162-7.

30. Smart NJ, White P, Allison AS, et al. Deviation and failure of enhanced recovery after surgery following laparoscopic colorectal surgery: early prediction model. Colorectal Dis 2012; 14 e727-34.

31. Lewis SJ, Andersen HK, Thomas S, et al. Early enteral nutrition within $24 \mathrm{~h}$ of intestinal surgery versus later commencement of feeding: a systematic review and meta-analysis. J Gastrointest Surg 2009; 13: 569-75.

Received: 17.12.2016, accepted: 2.03.2017. 